\title{
Hyperandrotrema cetorbini n. g. n. sp. (Trematoda, Sanguinicolidae) parasite du système circulatoire de Cetorbinus maximus (Selacii)
}

\author{
par C. MAILLARD * et M.-H. KTARI * *
}

* Laboratoire de Parasitologie comparée,

Université des Sciences et Techniques du Languedoc, F 34060 Montpellier Cedex.

***Faculté des Sciences. Unité de Biologie et de Physiologie animale.

Campus Universitaire El Menzah, Tunis, Tunisie.

\section{Résuméé}

Description d'un nouveau genre de Sanguinicolidae, Hyperandrotrema parasite d'un Cetorhinus maximus pêché sur les côtes tunisiennes.

Ce nouveau genre, placé dans la sous-famille des Chimaerohemecinae, se caractérise par un œsophage court et de longs caecums digestifs en forme de « $U$ » renversé.

La diagnose de la sous-famille des Chimaerohemecinae est amendée.

\section{Summary.}

Hyperandrotrema cetorhini n. g. n. sp. (Trematoda, Sanguinicolidae) parasite of the circulatory system in Cetorhinus maximus (Selachii).

Description of a new genus of Sanguinicolidae, Hyperandrotrema from one Cethorhinus maximus captured on Tunisian coasts.

This new genus included in the subfamily of Chimaerohemecinae is characterized by a short gullet and long digestive caeca shaped like an inverse « $U$ ».

Thus, the diagnosis of the subfamily of Chimaerohemecinae is modified.

Accepté le 3 février 1978. 
Au mois de mars 1976, un Requin Pèlerin, Cetorhinus maximus (Gunnerus, 1765) fut capturé au filet trémail, à Teboulka sur les côtes tunisiennes. Ce Sélacien était un individu mâle de 5,5 mètres de longueur totale.

La dissection de cet animal, en vue de recherches sur son helminthofaune, a mis en évidence une très grande quantité de Trématodes dans le système circulatoire. Ces parasites, appartenant à une famille des Sanguinicolidae Graff, 1907, étaient présents dans la totalité des vaisseaux sanguins et extrêmement abondants dans le cœur. Cependant, aucun organe ne présentait de malformations dues à ce parasitisme.

Nous donnons ci-dessous une description de ce Digène.

\section{Hyperandrotrema cetorbini n. g. n. sp.}

Hôte : Cetorhinus maximus (Gunnerus, 1765).

Habitat : Système circulatoire.

Localité : Côtes tunisiennes.

Matériel étudié : 35 individus colorés au Carmin boracique et montés in toto: 6 individus débités en coupes sériées transversales et colorés au Glychemaluméosine.

Type et cotype déposés au Museum National d'Histoire Naturelle de Paris sous les $\mathrm{n}^{\circ s}: 68$ PE TJ 19 et 68 PE TJ 20.

Mensurations (moyennes calculées sur 10 individus montés in toto) :

Longueur du corps: $6,670 \mathrm{~mm}(5,140 \mathrm{~mm}-7,500 \mathrm{~mm})$.

Largeur du corps : $2,970 \mathrm{~mm}(2,060 \mathrm{~mm}-3,910 \mathrm{~mm})$.

Cavité orale : $0,240 \mathrm{~mm}(0,200 \mathrm{~mm}-0,310 \mathrm{~mm})$.

Longueur du testicule : 4,140 mm (3,060 mm-5,780 mm).

Largeur du testicule : 2,380 mm (1,670 mm-3,400 mm).

Longueur de l'œsophage: $0,890(0,500 \mathrm{~mm}-1,870 \mathrm{~mm})$.

Longueur des épines : $10 \mu \cdot \mathrm{m}(9 \mu \mathrm{m}-12 \mu \mathrm{m})$.

\section{Description.}

Morphologie (fig. 1).

Ce trématode de grande taille est de forme ovoïde légèrement aplati dorsoventralement. Il ne possède ni ventouse orale ni acetabulum. Les téguments minces sont dépourvus d'épines sauf au niveau de deux bandes latéro-ventrales. Celles-ci présentent de chaque côté, sur toute la longueur du corps, 3 à 4 rangées longitudinales d'épines. Ces épines ont la forme d'un crochet dont la base bifide est aplatie dans un plan perpendiculaire à celui de la lame. 
ANATOMie (fig. 1 et 2 ).

L'appareil digestif. La bouche antérieure et sub-apicale se continue par une cavité sphérique à paroi non musculaire où débouchent les canaux de très nombreuses glandes unicellulaires. Après un court œsophage, l'appareil digestif se bifurque en deux branches à lumière régulière qui atteignent la partie postérieure du corps où elles se terminent en caecums aveugles. Au niveau de la poche du cirre, la branche digestive gauche effectue une anse dorsale pour contourner cet organe.

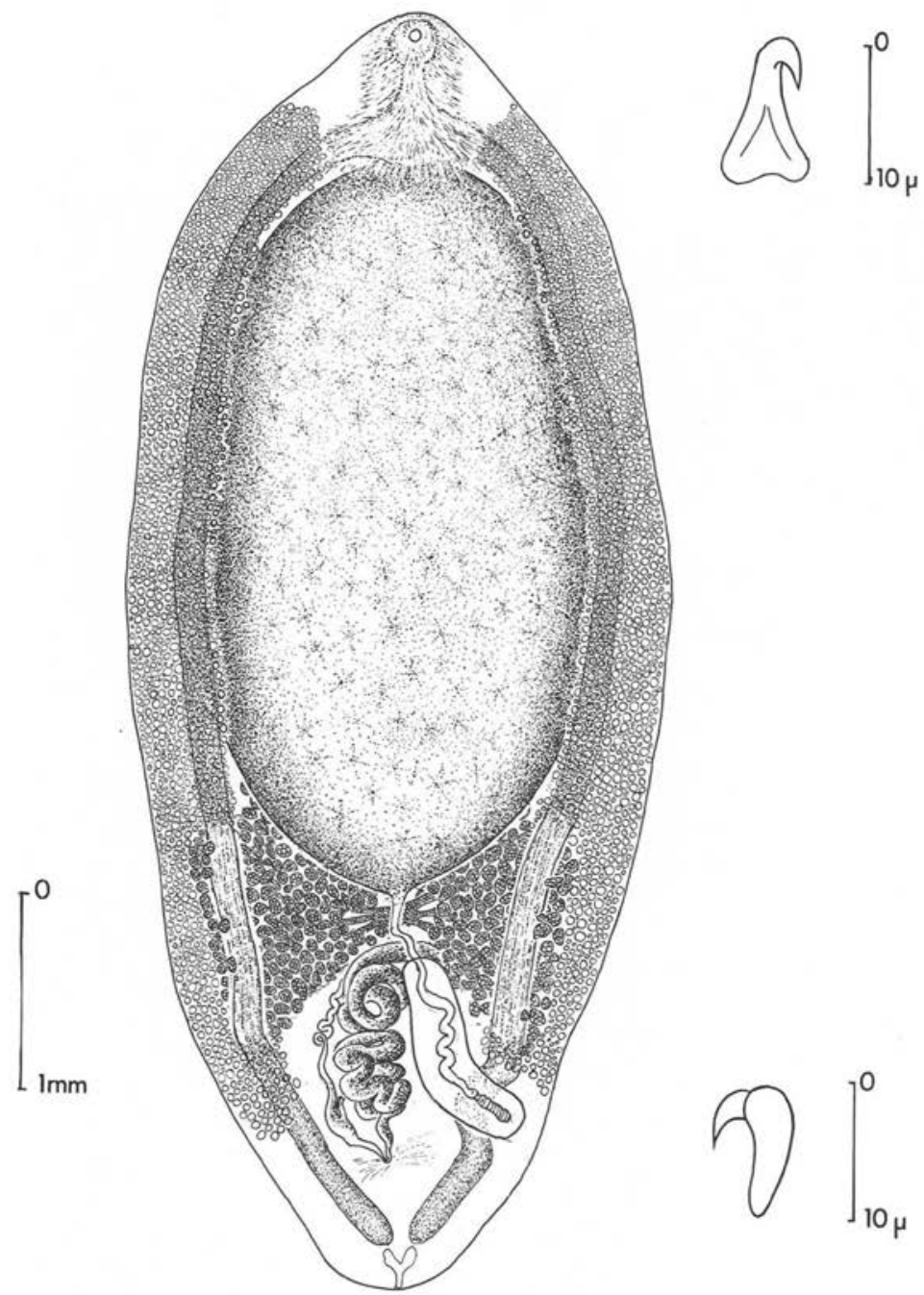

Fig. 1. Hyperandrotrema cetorhini n. g. n. sp. animal in toto, en vue ventrale. 
L'appareil génital mâle. Le testicule unique par son développement extraordinaire est caractéristique de l'animal. En effet, cet organe, bien individualisé, représente les deux tiers de la longueur totale. Il débute juste après la bifurcation intestinale, occupe tout l'espace intercaecal et se termine au tiers postérieur du corps. Il en part sur le plan médian, un court canal déférent à paroi mince et à la lumière large. Ce canal déférent aboutit à une grande poche du cirre placée sur le côté gauche du corps par rapport au plan médio-sagittal de l'animal. A l'intérieur de cette poche, le canal déférent se dilate légèrement en une vésicule séminale interne flexueuse à laquelle fait suite un cirre évaginable à paroi musculaire.

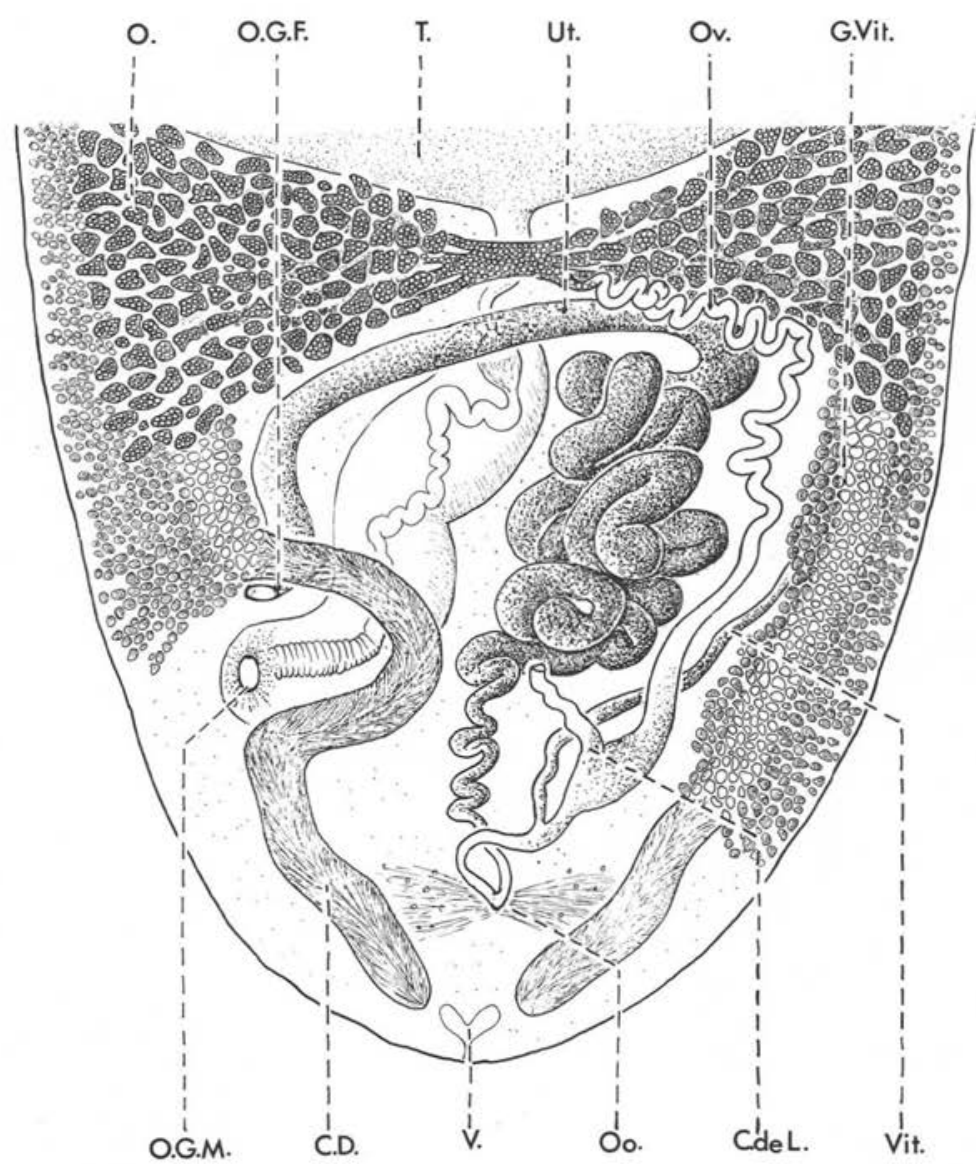

Fig. 2. Hyperandrotrema cetorhini n. g. n. sp. extrémité postérieure de l'animal en vue dorsale. C.D.: Canal déférent ; C. de L. : Canal de Laurer; G. Vit.: Glandes vitellogènes ; O.: Ovaire; O.G.F.: Ouverture génitale femelle; O.G.M.: Ouverture génitale mâle ; Oo: Ootype ; Ov. : Oviducte ; T. : Testicule ; Ut. : Utérus ; V. : Vessie ; Vit. : Vitelloducte. 
L'ouverture de la poche du cirre est située latéro-dorsalement au fond d'une légère dépression, sur le côté gauche du corps.

L'appareil génital femelle. L'ovaire est placé immédiatement en arrière du testicule dont il recouvre parfois dorsalement le bord postérieur. Il est formé de deux arborescences latérales, réunies sur le plan médian.

De cette région centrale part, dorsalement, un oviducte très sinueux qui descend vers la région postérieure en longeant la branche digestive droite. Il s'élargit, donne le canal de Laurer avant de reprendre son diamàtre initial.

Cet élargissement pourrait correspondre à un réceptacle séminal, mais nous n'y avons jamais observé de spermatozoïdes. L'oviducte effectue ensuite une anse postérieure contenant l'ootype entouré par les glandes de Mehlis.

Le canal de Laurer, court, débouche au milieu de la face dorsale au niveau de l'ouverture génitale mâle.

Les glandes vitellogènes formées de très nombreux follicules de petite taille, entourent les caecums digestifs. Elles débutent au niveau de la bifurcation des branches digestives et se terminent peu après l'ovaire sur le côté gauche et un peu plus en arrière du côté droit.

Après l'ootype, l'utérus remonte en décrivant de très larges boucles situées légèrement à droite du plan sagittal. Au niveau du pont ovarien, l'utérus passe sur le côté gauche du corps dorsalement par rapport au canal déférent et la poche du cirre. Il redescend ensuite le long de la branche digestive gauche et se termine dorsalement en avant de l'ouverture mâle.

Nous n'avons que très rarement observé des œufs dans l'utérus et celui-ci était entièrement rempli de cellules vitellines plus ou moins dégradées. Les quelques œufs rencontrés, avaient une forme sub-sphérique irrégulière et paraissaient teratologiques.

L'appareil excréteur est représenté par une très petite vessie à paroi mince et en forme de « $Y$ » s'ouvrant à l'extrémité postérieure du corps.

\section{Discussion}

Par son anatomie, ce Trématode doit être rangé dans la famille des Sanguinicolidae Graff, 1907.

En 1971, dans son «Synopsis of Digenetic Trematodes of Vertebrates » Yamaguti créait la sous-famille des Chimaerohemecinae pour le genre Chimaerohemecus Van der Land, 1967.

Il proposait pour la sous-famille la diagnose suivante :

«Corps cylindrique légèrement aplati, inerme, pas de ventouse orale. Esophage très long entouré par des cellules glandulaires. Caecums en forme de U inversé, longs, s'étendant en arrière de l'ovaire. Testicules divisés en de nombreux follicules, intercaecaux et pré-ovariens. Poche du cirre présente, contenant une vésicule séminale 
cylindrique et un cirre. Ouvertures génitales mâle et femelle séparées près de la terminaison du caecum digestif droit. Ovaire médian multilobé à une courte distance en arrière du plan équatorial. Oviducte descendant, se réunissant au vitelloducte dans la partie postérieure du corps. Canal de Laurer présent. Réceptacle séminal absent. Utérus flexueux, dans le champ médian entre les glandes coquillières et l'ovaire, s'ouvrant juste au-dessus de l'ouverture mâle. Glandes vitellogènes s'étendant dans les champs latéraux entre l'extrémité antérieure et les extrémités caecales. Vésicule excrétrice en forme de V. »

Toutefois, la diagnose proposée par Yamaguti présente certaines contradictions avec la description donnée par Van der Land (1967) de l'unique genre de cette sous-famille.

En effet, Chimaerohemecus Van der Land, 1967 possède des épines recourbées, situées sur les bords ventraux du corps et n'est donc pas inerme. Son testicule est unique et non folliculaire. Le réceptacle séminal est présent. Enfin, selon la description donnée par Van der Land, les ouvertures génitales sont placées à gauche de l'animal et non à droite comme le note Yamaguti.

Compte tenu de ces rectifications, l'espèce que nous venons de décrire correspond presque complètement à la diagnose de la sous-famille.

Cependant, le Trématode découvert chez Cetorhinus maximus diffère de Chimaerohemecus trondheimensis Van der Land, 1967 par la longueur très réduite de son œsophage et par le développement des caecums digestifs qui atteignent la région postérieure du corps.

Chez les Sanguinicolidae, la structure de l'appareil digestif est importante au point de vue systématique. Nous pensons donc que le parasite de Cetorhinus doit être placé dans un nouveau genre que nous proposons de nommer Hyperandrotrema n.g. et qui aura la diagnose suivante :

Corps ovoïde aplati dorso-ventralement. 2 rangées longitudinales d'épines latéroventrales. Pas de ventouse orale ni d'acetabulum. Esophage très court. Caecums digestifs en forme de $U$ inversé se terminant au niveau de l'extrémité postérieure du corps. Testicule unique, de grande taille. Poche du cirre s'ouvrant latéro-dorsalement dans le quart postérieur gauche. Ovaire post-testiculaire. Canal de Laurer présent. Ootype postérieur. Utérus entièrement post-ovarien. Ouverture génitale femelle dorsale et gauche, distincte de l'ouverture génitale mâle. Glandes vitellogènes folliculaires entourant les caecums digestifs. Vessie en forme de Y.

Espèce-type : Hyperandrotrema cetorhini n. sp.

Nous plaçons ce genre dans la famille des Sanguinicolidae Graff, 1907 et la sous-famille des Chimaerohemecinae Yamaguti, 1971 dont nous amendons la diagnose de la manière suivante:

Corps cylindrique légèrement aplati. 2 rangées d'épines latéro-ventrales. Pas de ventouse orale ni d'acetabulum. Esophage de taille variable entouré par des cellules glandulaires. Caecums en forme de U inversé, longs, se terminant soit en arrière soit plus en avant au niveau de l'ovaire. Testicule unique. Poche du cirre présente à ouverture postérieure latéro-dorsale. Ovaire plurilobé post-testiculaire. 
Canal de Laurer présent. Ouverture génitale femelle distincte de l'ouverture génitale mâle, toutes deux placées à gauche. Glandes vitellogènes folliculaires entourant les caecums digestifs. Vessie en forme de V ou de Y.

Parasite du système circulatoire des Chimères et des Sélaciens.

Genre-type : Chimaerohemecus Van der Land, 1967.

Cette différence d'hôte est à noter car les chimères sont des animaux benthiques alors que le Requin-Pèlerin est un Poisson pélagique. Le cycle évolutif de ces Trématodes doit montrer des modalités différentes.

De plus, malgré le grand nombre d'individus présents dans les vaisseaux et le cœur du Requin-Pèlerin que nous avons examiné, aucun de ces parasites ne présentait d'œufs dans l'utérus et les rares œufs observés semblaient tératologiques.

Dans sa description de Chimaerohemecus trondheimensis, Van der Land note dans l'utérus de cette espèce la présence d'œufs fortement déformés. Cette absence d'œufs normaux est assez remarquable car les Sanguinicoles, au contraire, montrent la plupart du temps une production d'œufs intense, sans doute pour pallier les difficultés de dispersion.

La biologie de ces parasites est malheureusement inconnue et il est possible qu'il existe un cycle de ponte annuel, ces animaux n'étant gravides qu'à certaines périodes de l'année.

\section{Bibliographie}

Van der Land J. (1967): A new blood fluke (Trematode) from Chimaera monstrosa L. Koninkl. Nederl. Akad. Vanwetenshappen Amsterdam Sér. C, 70, 110-120.

Yamaguti S. (1971): Synopsis of Digenetic Trematodes of Vertebrates. Keigaku Publi. Co.. Tokyo, 1074. 